\title{
Flaws in the Literature
}

\author{
Wagner HL* \\ Professor of Family medicine, Masters in Science, Curitiba, Brazil
}

*Corresponding author: Hamilton Lima Wagner, MD, Masters in Science, Curitiba,

Brazil; Email: hamiltonw@uol.com.br

\section{Editorial \\ Volume 2 Issue 2}

Received Date: March 11, 2019

Published Date: March 14, 2019

DOI: $10.23880 /$ jqhe- 16000116

\section{Editorial}

In 1986 a family physician, Marc Jamoulle, et al. [1] coined the idea of quaternary prevention. It was another era, and the perspective we face at that moment was that we would be able to offer to our patient's good care without harm with excessive intervention. It remains, but the problem now is even bigger.

We are now dealing with two new situations, first we have patients that are over informed about health care, and influenced by the media, which almost ever is developed and founded by the health industry. Second we have safety problems with medical research, also founded by the health industry and developed in a way that it is not available to independent analysis.

It is now time to reflect how to develop an approach to medical practice considering the principles of quaternary prevention in this new scenario. There must be another form to talk and to share decision with this population that challenges us with their information distorted by the media.

The internet is a very interesting tool to communicate, but it has no filter the information is listed by relevance, but distorted by commercial fees. And the reader frequently does not have a background to understand and interpret this data. This leads to uncertainty and misunderstand, creating anxiety and a need for lots of tests and interventions.

Medicine is running on a path that leads to the biological comprehension of all facts, but this does not solve many situations that we face in clinical practice. Life is not only a biological sequence of events; it is changed by the environment and social behavior. And that is the challenge that must be faced if there are expectations to offer quality of life and increase health perception.
There are some concepts that cannot be missed, and unfortunately they are often shadowed by the press, who use it to launch stories that are far from the truth. Life has duration, and besides, it has been prolonged in the last two centuries, mostly because of social conditions, not because of medical intervention. We could say that medical science contributed just $10 \%$ to life expectancy [2].

The introduction of the concept of quaternary prevention - nothing more than the systematization of the concept of "primum non nocere" in our modern world and medical practice is an ethical approach to practicing a better clinical care and to protect people from an excess of medicine $[3,4]$. But it could be the beginning of a new paradigm in modern medicine, where a person's life is at the center of the care and it has been developed to offer the best standard we can offer to that person in their own milieu.

The concept of quaternary prevention is a very central and severe warning in literature problems, the flaws in the literature leads to malpractice, even if the caregiver is very committed to good practice.

It is necessary to think how is the evidence produced in the actual medical method, and what are the results that can be achieved with this information. And we must be aware that information is something very different from knowledge.

The information released in the last 30 years had made profound changes in medical care; the amount of research published every year is astonishing. But the center of all these research is barely direct to common people and their problems, most of the data are to change the way health is defined and almost all decrease standards that leads to more intervention, without any 


\section{Journal of Quality in Health Care \& Economics}

significant change in life expectancy but increasing costs and suffering.

This problem appeared to be well addressed when Sackett, et al. [5] coined the concept of evidence based medicine, which tackle the need for good research evidence with excellent expertise of the physician in the care of patients. But it does not occur that way. There are misconducts in medical research and practice, influenced by a biased health industry interested in producing money.

The first big gap that must be faced is to find and publish the research which was hidden. It must be made clear to everyone that unfavorable results are just as important to make a good decision. It could even protect people from exposure to treatments that have been proven harmful [6].

It is common run several researches in a field, and then select the data that could be of interest - and simply hide the others. This kind of flaw leads to an incorrect comprehension of the data, and perhaps to do harms that could easily be avoided. The knowledge of these data could allow physicians to recognize side effects, and lack of results [6].

The second step is to review every protocol of the research. Effective research must try to prove that the hypothesis is false, and that is not what can be found when the protocols are open often the researcher used drugs that were inadequate, in order to say that the tested one was better. Statistics is a science that can easily show results that are expected, and the only security that we have is to find the way the trial was designed in order to identify weakness and the intention of the research.

The way a research is developed can show a lot about it's own strength, and how could this affect clinical practice. But when this data were accessed by independent researchers what was found is that there are many weakness, the criteria to select the sample is not very well defined and, worst, some exclusion criteria may be create after the research to support a good result. There are many fines applied to pharmaceutical companies about miss conduction of their research and it must be fixed [7].

The third step is to look at the raw data, by independent researchers, to identify clearly in which population and in which conditions the research was done. This act identify the conditions that were missed in the file published, and will gave transparency to it, offering a more reasonable understanding of the article.

The Cochrane Collaboration, an independent organization that works reviewing researches and producing analysis about them can't access most of the data that supports the published research, creating uncertainty even after an analysis. This is a major problem. And even this institution had been flawed by the health industries.

Many authors have published papers on these issues and it is time to reflect what is important to a good medical practice [8]. We have now settled standards, based not in quality and quantity of life, but in laboratory research which develop standards applied to ideal people. Many of these standards had been narrowed along the time, not for a better care but in the interest of the health industry. As an example the first definition of depression settled that there was no condition to provoke the feelings of unhappiness, now that clause was abandon.

The researchers have narrowed definitions over hypertension, diabetes, dyslipidemia, and many other situations. They have compared standards of young people with older ones and pushed the treatment to restore a condition that wasn't possible anymore [9-11]. These definitions had led to a very aggressive intervention, and an abuse in the use of technology and medicines.

In the path to a shared decision, it is important that everybody believes on the literature, and it is fundamental to consider that, even documents, like AGREE II and GRADE that try to protect physicians and patients $[12,13]$. However there is still a lot of pressure to do practices that are not in conforming with the concepts of quaternary prevention. We are still developing medical interventions that are not in benefit of the patients, but on the interest of the health industry [14].

The research on quality is on their way, but it's essential an ethical change in the meanings of medicine thinking we must focus on patients and their families, not in science or profits [15].

\section{References}

1. Jamoulle M (1986) Information and computerization in general medicine. University Presses of Namur, pp: 193-209. 


\section{Journal of Quality in Health Care \& Economics}

2. Mendes EV (1996) A schedule for the health. Hucitec, São Paulo.

3. Jamoulle M, Berstein J, Pizzanelli Baez M, Silva A, Wagner H (2014) Quaternary prevention: a concept based on time and relationship. Second part: the astonishing international diffusion of the concept. Médecine Fev 10(2): 75-77.

4. Jamoulle M (2015) Quaternary prevention, an answer of Family doctors to over medicalization. Int J Health Policy Manag 4(2): 61-64.

5. Sackett DL (1997) Evidence-based Medicine. Churchill Livingstone, New York.

6. Goldacre B (2013) Bad pharma: how drug companies mislead doctors and harm patients. Faber and Faber, New York.

7. Ioannids JPA (2005) Why most published research findings are false. PLoS Med 2(8): e124.

8. Gotsche PC (2013) Deadly medicines and organized crime, how does big pharma has corrupted health care. Internal Medicine Journal 44(8): 310.

9. Every Palmer S, Howick J (2014) How evidence-based medicine is failing due to biased trial and selective publication. J Eval Clinl Pract 20(6): 908-914.
10. Greenhalg T, Howick J, Maskrey N (2014) Evidence based medicine: a movement in crisis? BMJ 348: g3725.

11. Akst J (2014) Dealing with irreproducibility. The Scientist.

12. Brouwers MC, Kho ME, Browman GP, Burgers JS, Cluzeau F, et al. (2010) Agree II: advancing guideline development, reporting and evaluation in health care. CMAJ 182(18): E839-E842.

13. Atkins D, Eccles M, Flottorp S, Guyatt GH, Harbour RT, et al. (2004) Systems for grading the quality of evidence and the strength of recommendations II: pilot study of a new system. BMC Health Serv Res 5(2): 25.

14. Wagner $H$ (2015) Quaternary Prevention and the Challenges to Develop a Good Practice; Comment on "Quaternary Prevention, an Answer of Family Doctors to Over medicalization" Int J Health Policy Manag 4(8): 557-558.

15. Torka M, Mintzes B, Bhasale A, Fabbri A, Perry L, et al. (2019) Unacceptable secrecy" around potential safety risks of medicines. 